\title{
Fluxo para Construção do Modelo de Velocidade Regional da Bacia de Campos
}

Frank Cenci Bulhões, Guilherme Antônio Sampaio de Amorim, Vera Lúcia do Vale Sales Roca Bruno, Gleidson Diniz Ferreira, Emmanoelle Santos Pereira, Rosângela Foletto de Castro; Petrobras

Copyright 2014, SBGt - Sociedade Brasileira de Geofísica

Este texto foi preparado para a apresentação no VI Simpósio Brasileiro de Geofísica, Porto Alegre, 14 a 16 de outubro de 2014. Seu conteúdo foi revisado pelo Comitê Técnico do VI SimBGf, mas não necessariamente representa a opinião da SBGf ou de seus associados. E proibida a reprodução total ou parcial deste material para propósitos comerciais sem prévia autorização da SBGf.

\section{Abstract}

The construction of the velocity model for time to depth conversion is one of the crucial steps in building the geological model. The important issue on creation of velocity model is the difficulty of data standardization due to complexity, heterogeneity of different data types, model dimension and resolution. This paper describes the flow to the construction of the regional velocity model in the Campos Basin. The process of analysis of data and modeling is done through the software SKUA-GOCAD (Paradigm Ltd). The results were compared to data from existing wells in the area and considered good.

\section{Introdução}

O geofísico para construir um modelo de velocidade deve ter em mente alguns pontos importantes, nos quais estão a ordem de grandeza, complexidade estrutural dos objetivos em sub-superfície, os tipos de dados e sua qualidade. A construção do modelo de velocidade para conversão de tempo para profundidade é uma das etapas de fundamental importância na construção do modelo geológico. Os fatores nos quais devem ser levados em consideração são: sua complexidade geológica, o tempo operacional e a especialização (algoritmos). Em se tratando de um modelo regional, no caso para a Bacia de Campos, nos deparamos com diversos desafios, nos quais estão: o limite computacional com volume de dado sísmico, diferentes tipos de velocidade e procedências, análises e filtragens estatísticas (Maul et al., 2013) para concatená-las e conformá-las, análise e filtragem de dados de poços e parâmetros de calibração. A área de nosso estudo abrange a Bacia de Campos, com aproximadamente $247.000 \mathrm{~km}^{2}$, sendo $515 \mathrm{~km}$ na direção norte-sul e $415 \mathrm{~km}$ leste-oeste (Fig. 1).

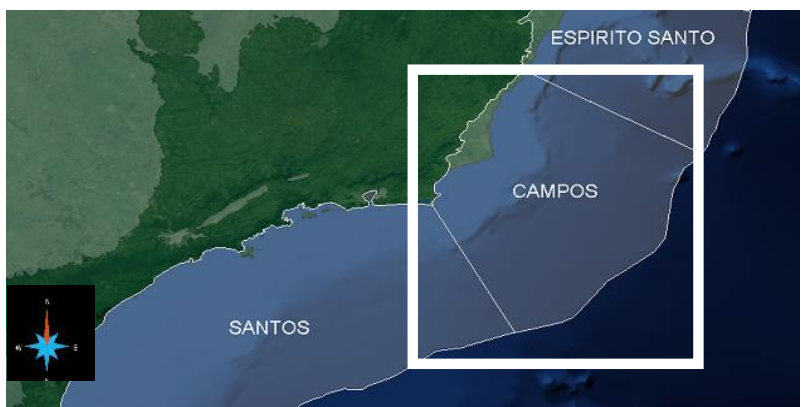

Figura 1 - Área da modelagem - Bacia de Campos

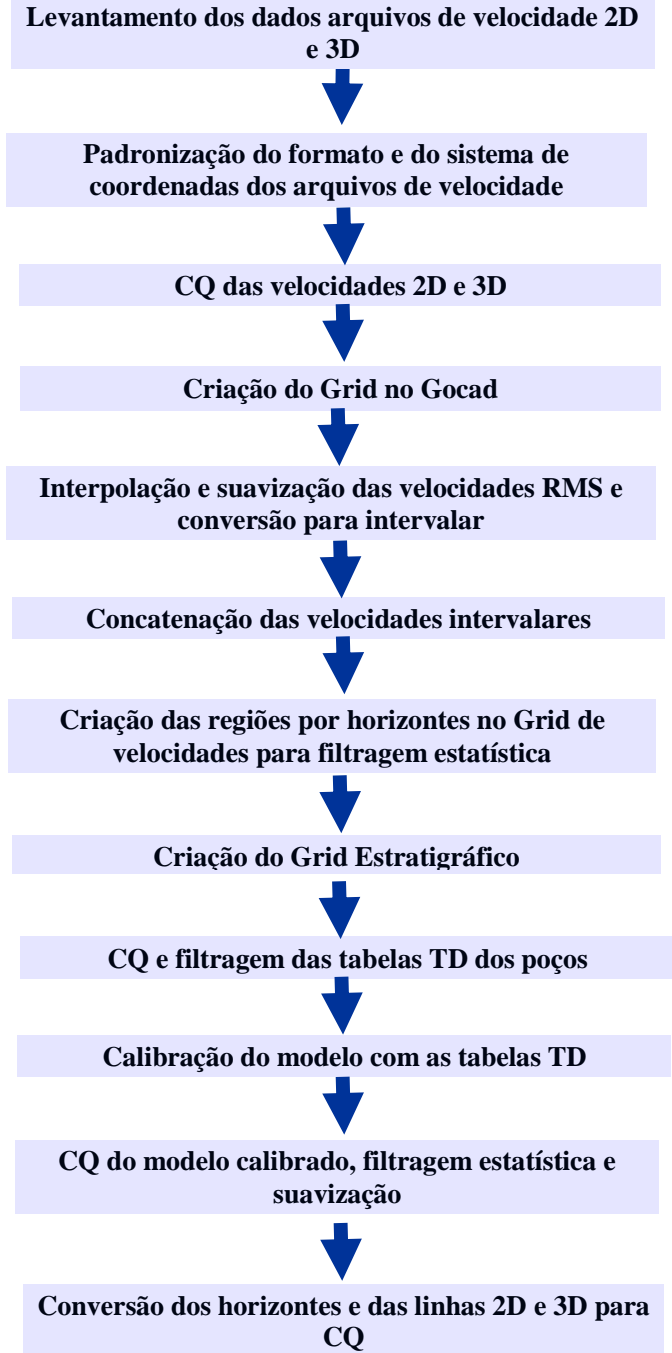

Figura 2 - Fluxograma para construção do modelo regional

\section{Metodologia de Trabalho}

\section{Limitação Computacional $X$ Resolução Sísmica}

O processo de modelagem foi realizado no software SKUA-GOCAD da Paradigm Ltd. Na Fig. 2 apresentamos o fluxo adotado. A escolha da malha do grid de um modelo depende da limitação computacional dos softwares envolvidos e a ordem de grandeza dos objetos estruturais na região de interesse. Quanto mais densa a 
malha, maior será o detalhamento. Por outro lado, a eficiência da performance computacional ficará comprometida. Um dado não adequadamente bem amostrado pode gerar perdas de informações estruturais significativas. A malha que adotamos foi de $1 \mathrm{~km} \mathrm{X1 \textrm {km }}$ $\times 25 \mathrm{~ms}$.

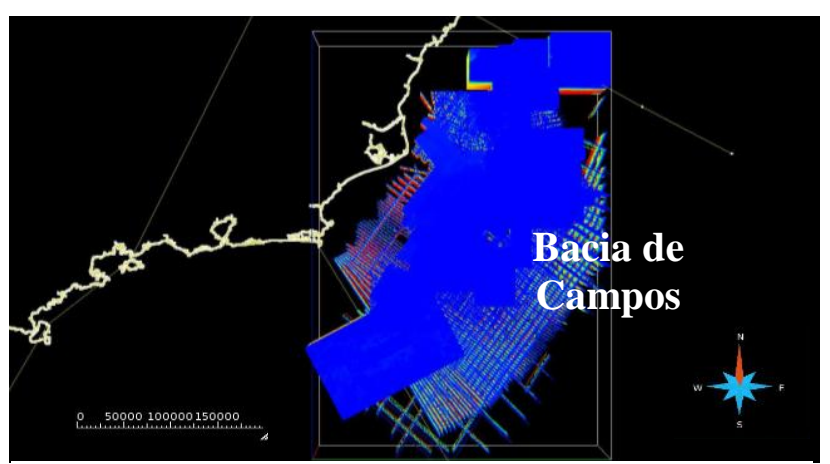

Figura 3 - Velocidades 2D e 3D vista em planta Bacia de Campos

\section{Tipos de Velocidade}

Para a modelagem em questão, utilizamos velocidades de diversas procedências, aquisições e processamentos. As velocidades envolvidas na construção do modelo foram: RMS 2D, RMS 3D, Migração PSTM 2D, PSTM 3D e PSDM 3D (Fig. 3) O desafio em concatenar essas diversas velocidades está em conformar lateralmente volumes que foram construídos utilizando malhas, algoritmos e parâmetros diferentes. Velocidades de migração em tempo são mais adequadas para imageamento de estruturas de pouca complexidade e variando verticalmente, por outro lado, a migração em profundidade é direcionada a estruturas com maior complexidade geológica estrutural (diápiros de sal) e para qualquer tipo de distribuição de velocidade. Para conformar essa diversidade de velocidades presentes na região, utilizamos 5 horizontes, com os quais criamos camadas para controle de qualidade, filtragem estatística e suavização. Em áreas onde ocorreram interseções dos volumes, consideramos como ordem de importância: PSDM 3D, PSTM 3D, RMS 3D, velocidades 2D.

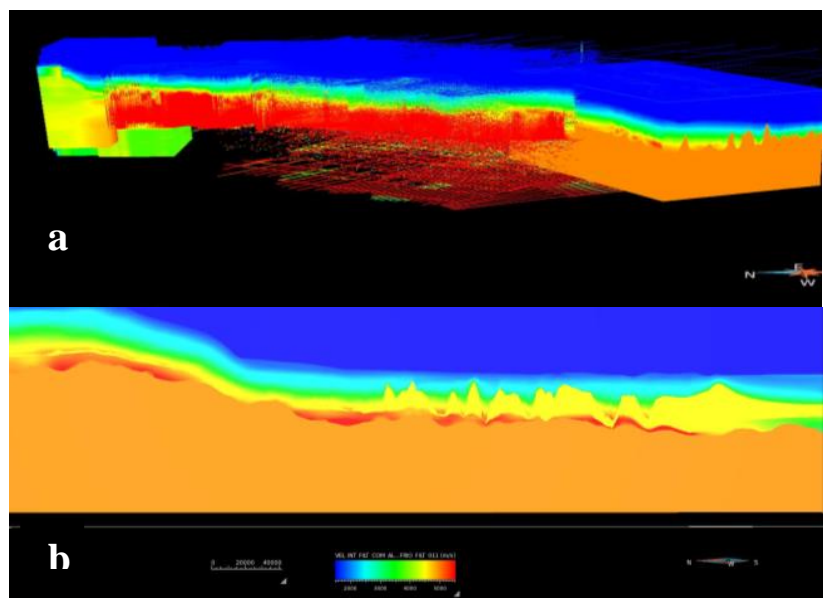

Figura 4 (a) Velocidades intervalares $2 D$ e $3 D$ originais. (b) Velocidades intervalares concatenadas, filtradas e suavizadas.

\section{Grid Estratigráfico}

Para uma melhor conformidade da estratigrafia, utilizamos a ferramenta de deformação da malha existente no Gocad-Skua, o Grid Estratigráfico A deformação do grid é proporcional aos horizontes e conforme a sua estrutura.

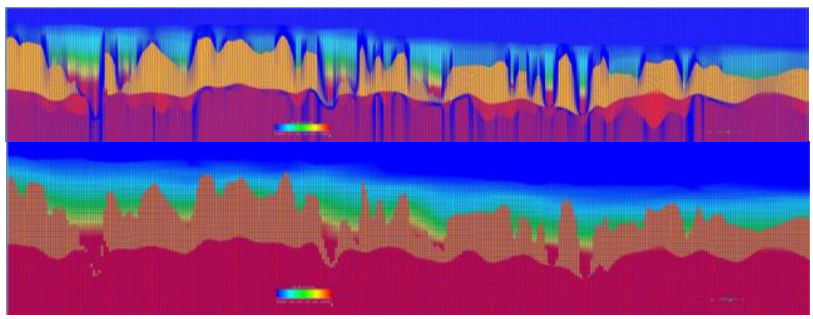

Figura 5 - Grid Estratigráfico (Gocad) X Grid Regular

\section{Dados de poços e calibração}

Um modelo de velocidade para migração PSDM tem como objetivo 0 imageamento dos refletores $e$ posicionamento das estruturas lateralmente, não necessariamente colocar os eventos em uma profundidade mais acurada. Para o ajuste do campo de velocidades para a conversão de tempo para profundidade utilizamos os dados de poços (tabelas tempo-profundidade). Foram utilizados 29 poços distribuídos ao longo da região de interesse. A calibração do modelo pelas tabelas tempo-profundidade filtradas foi realizada utilizando krigragem com deriva externa, utilizando a elipse de anisotropia com eixo maior na direção do trend dos poços (Fig. 6) no grid estratigráfico (Fig. 7), escolha essa feita após testar outras direções.

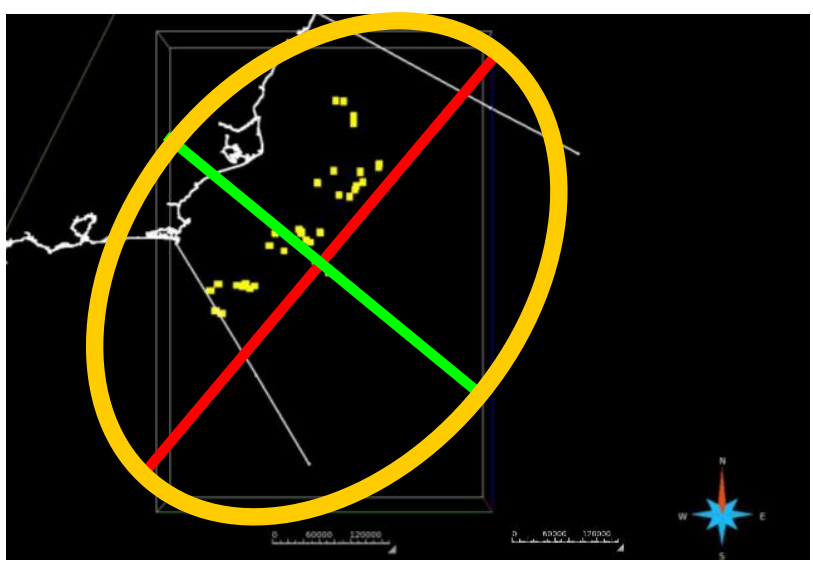

Figura 6 - Elipse de anisotropia para calibração do modelo de velocidade pelas tabelas tempo profundidade dos poços.

\section{Resultados e Discussões}

A partir do modelo calibrado (Fig. 7), convertemos os mapas dos horizontes e linhas sísmicas 2D e 3D. 


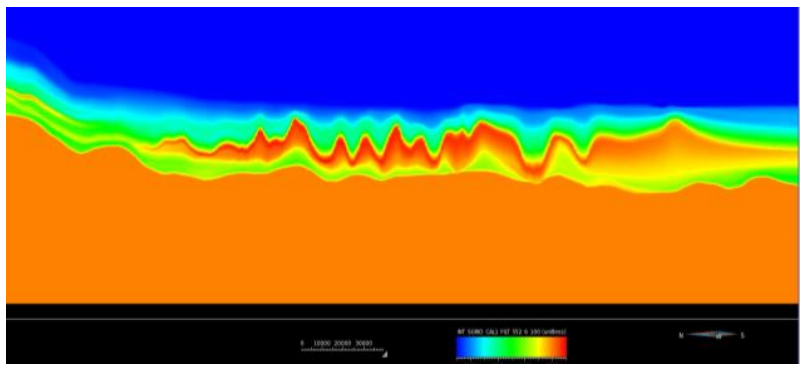

Figura 7-Modelo de Velocidade calibrado com as tabelas tempo-profundidade

Os mapas convertidos para profundidade estão apresentados na Figura 8. Fazendo-se uma análise qualitativa dos mapas convertidos observam-se realces e mudanças significativas nas estruturas comparadas aos mapas em tempo. O mesmo podemos observar nas seções sísmicas $2 D$ e $3 D$ nas figuras 9 e 10 respectivamente. Por outro lado, analisando-se quantitativamente os dados convertidos, observou-se um erro percentual da ordem de até $10 \%$, em profundidades entre 5000 e $6000 \mathrm{~m}$, comparados aos marcadores dos poços. Mediante as incertezas relacionadas à amostragem lateral, $500 \mathrm{~m}$, e vertical em torno de $80 \mathrm{~m}$ (critério de Widess) o modelo apresentou-se eficaz para obtermos as estruturas geológicas com razoável acurácia comparadas à ordem de grandeza, heterogeneidade dos dados e anisotropia.

\section{Conclusões}

O fluxo para a modelagem de velocidades para obtenção dos mapas em profundidade apresentado nesse trabalho proporcionou resultados satisfatórios diante dos desafios enfrentados. O objetivo desse trabalho foi um imageamento estrutural em profundidade. A predição de valores de profundidade mais acurados requer uma malha mais densa. Este modelo será aperfeiçoado com outros parâmetros de filtragem e calibração, incorporando-se novos dados de poços, sísmicas e horizontes.

\section{Agradecimentos}

Agradecemos à PETROBRAS por permitir a publicação deste trabalho.

\section{Referências}

ETRIS, E. L., CRABTREE, N. J., DEWAR, J., 2001. True Depth Conversion: More than a pretty picture. CSEG Recorder.

JONES, I. F. \& DAVISON, I., 2013. Seismic Imaging in and around salt bodies: problems and pitfalls. $75^{\text {th }}$ EAGE Conference \& Exhibition incorporating SPE EUROPEC 2013.

MAUL, A., ET AL, Modelagem Geológica de Velocidades Sísmicas Utilizando o Gocad (Maio 2013) - Material interno Petrobras.
JONES, I., 2008. SPG Hyderabad Key-Note Adress: Nonuniqueness and ambiguity in velocity model building. $7^{\text {th }}$ International Conference \& Exposition on Petroleum Geophysics

SKUA-GOCAD User Guide, 2013 Paradigm Ltd.

YILMAZ, O., 2001, Seismic data analysis: SEG.

XU, W. et al. 1992. Integrating Seismic Data in Resevoir Modeling: The Collocated Cokriging Alternative. $67^{\text {th }}$ Annual technical Conference and Exhibition of the Society of Petroleum Engineer.

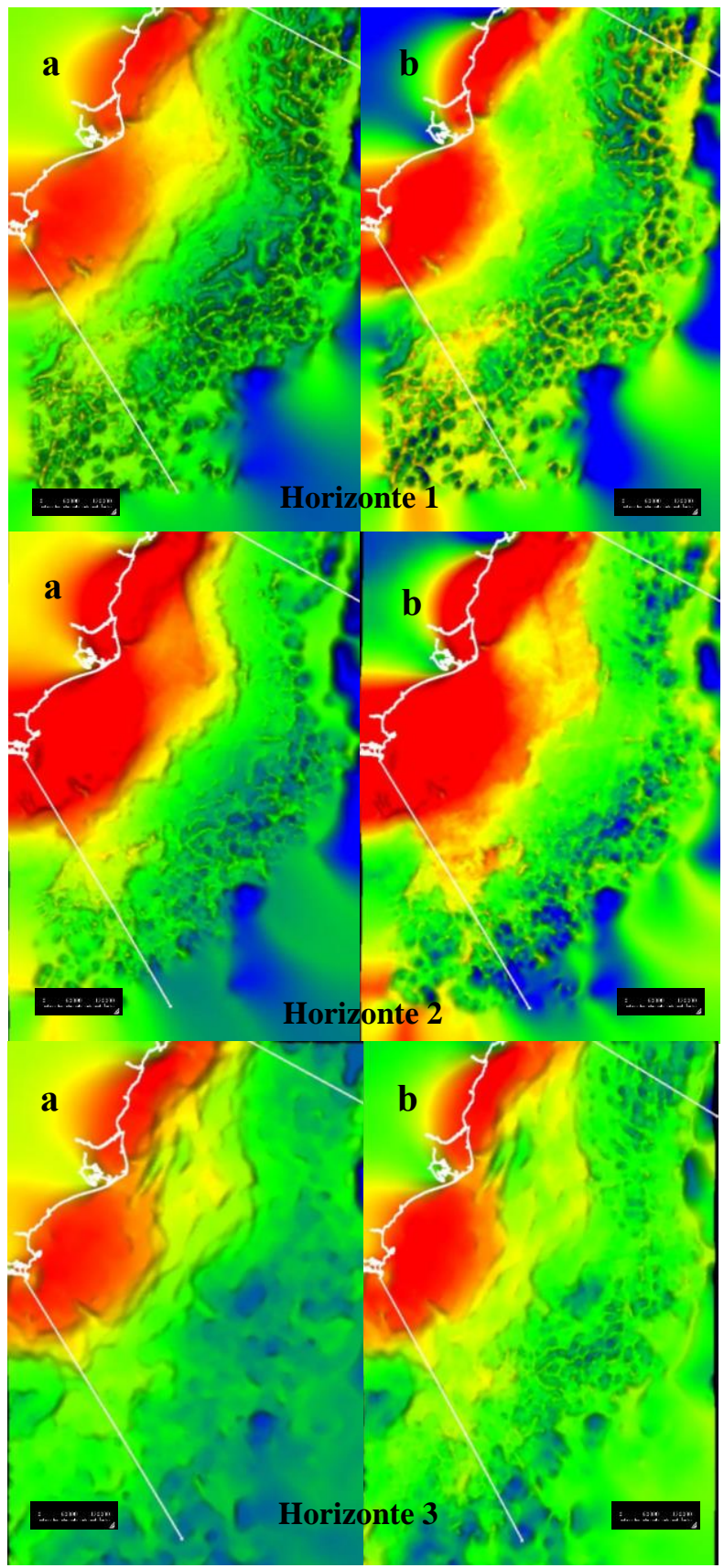

Figura 8- Horizontes 1,2 e 3 em tempo (a) e convertidos para profundidade (b) pelo modelo calibrado 


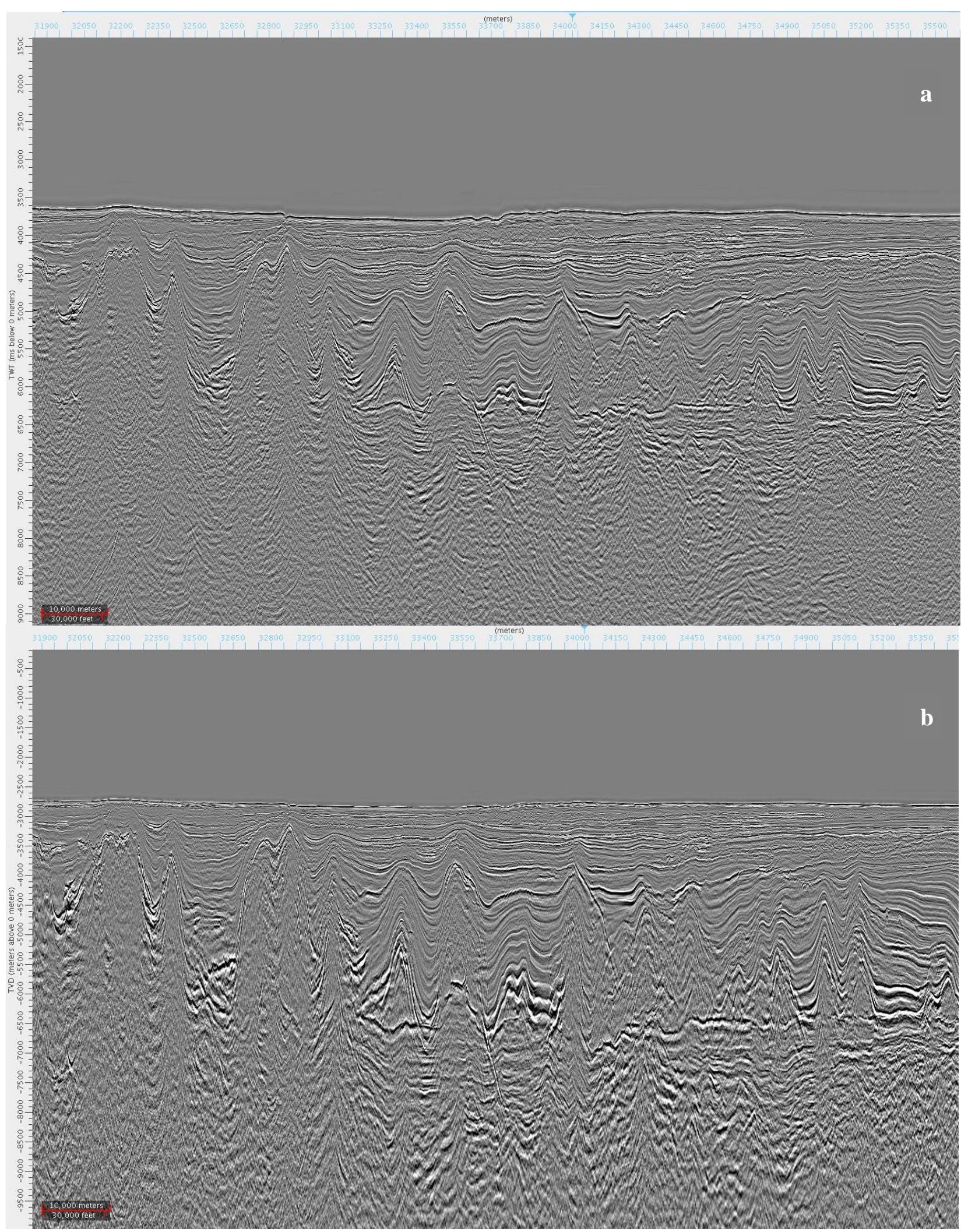

Figura 9- Linha sísmica 2D em tempo (a) e convertida para profundidade (b). 


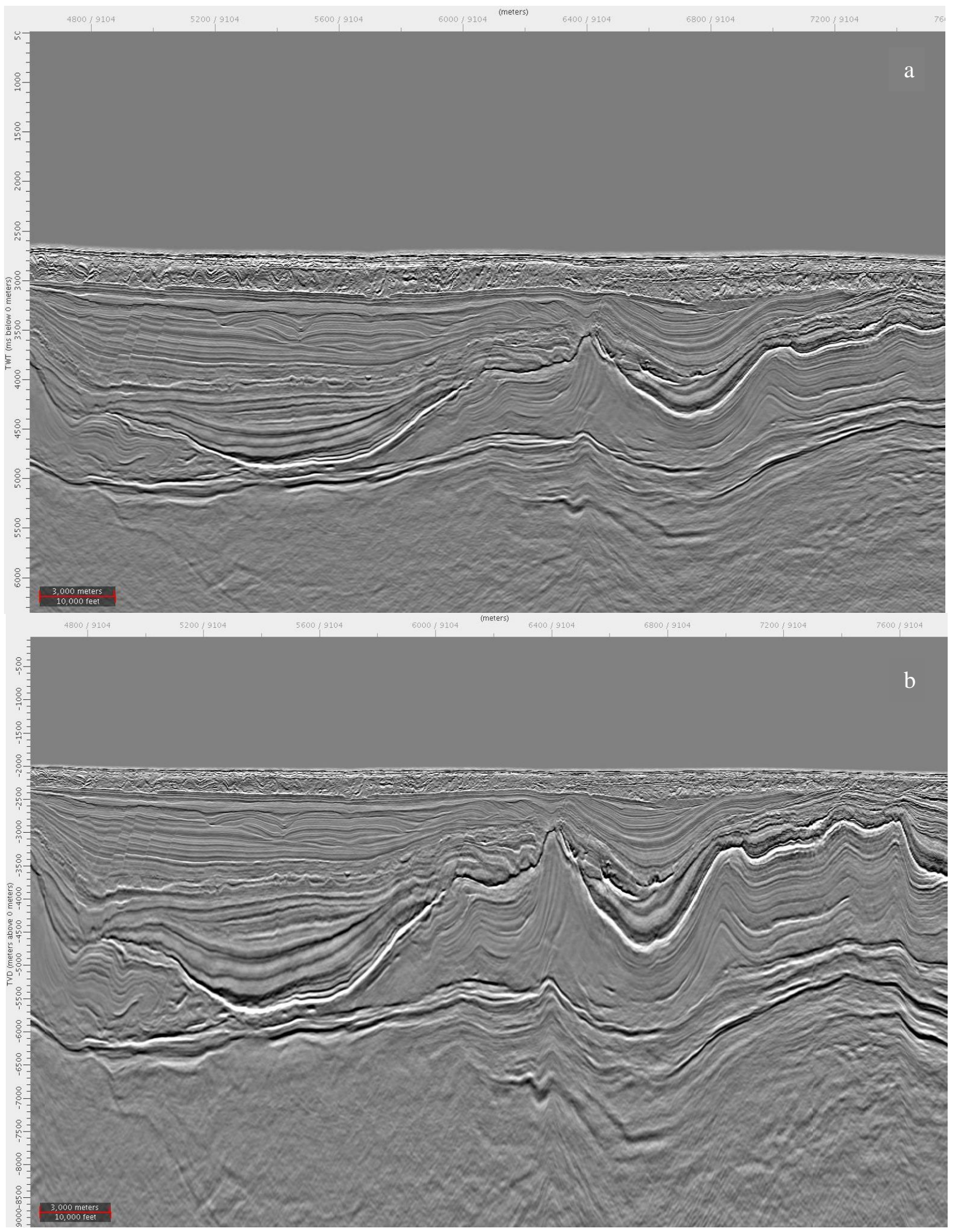

Figura 10 - Linha sísmica 3D em tempo (a) e convertida para profundidade (b). 